Original Article

\title{
HEMATOBIOCEMICAL AND HISTOPATHOLOGICAL STUDIES ON THE EFFECTS OF RAJ NIRWAN BATI (A NOVEL ALLOVEDIC DRUG) IN WISTAR RATS
}

\author{
AMITA SINGH $^{*}$ (iD , RAJ KUMAR ${ }^{2}$, SANJAY KUMAR KANNAUJIA ${ }^{3}$, NARESH PAL SINGH ${ }^{4}$
}

${ }^{1}$ Veterinary Surgery and Radiology, Veterinary Officer and In-Charge, Department of Animal Husbandry, India, ${ }^{2}$ Department of Neurosurgery, Former Vice-Chancellor, UP University of Medical Sciences and Head, SGPGIMS, Lucknow, India, ${ }^{3}$ Department of Pathology, UP University of Medical Sciences and Head, Department of Neurosurgery, SGPGIMS, Lucknow, India, ${ }^{4}$ Department of Community Medicine, UP University of Medical Sciences and Head, Department of Neurosurgery, SGPGIMS, Lucknow, India

"Email: amita.vet2020@gmail.com

Received: 21 Aug 2021, Revised and Accepted: 24 Dec 2021

\section{ABSTRACT}

Objective: To see the effects of Raj Nirwan Bati (RNB) on the hematobiochemical parameters, coagulation tests, and histopathological changes in the lungs, liver, kidneys and spleen and also to evaluate the immunomodulatory activity of RNB in Wistar rats.

Methods: A total of 24 adult albino Wistar rats (of bodyweight 200-250 g) of either sex were divided into 3 groups. In the normal control group ( $\mathrm{n}=8)$, no drug was administered and in the rest of the groups (A and B), $R N B @ 26 \mathrm{mg} / \mathrm{kg}$ body weight./day and $260 \mathrm{mg} / \mathrm{kg}$ body weight/day respectively were administered orally for a period of $14 \mathrm{~d}$. The blood samples were collected from the jugular vein at zero d (before drug administration) and after the $14^{\text {th }} \mathrm{d}$ of drug administration in both groups (A and B). The organ samples (lungs, liver, kidneys, and spleen) were collected after euthanizing the rats using Ketamine anesthesia overdose intraperitoneally (IP) after the $14^{\text {th }} \mathrm{d}$ of drug administration. White Blood Cells (WBC), Red Blood Cells (RBC), Hemoglobin (Hb), Hematocrit (HCT), Mean Corpuscular Volume (MCV), Mean Corpuscular Hemoglobin(MCH), Mean Corpuscular Hemoglobin Concentration(MCHC), number of platelets, Differential Leucocyte Count(DLC) i.e. the percentage of neutrophils, lymphocytes, eosinophils, monocytes and basophils, neutrophil adhesion percentage, Prothrombin test (PT), Activated Partial Thromboplastin Time (APTT), fibrinogen, D-dimer, Lactate Dehydrogenase (LDH), urea, creatinine, Aspartate Amino Transferase (AST), Alanine amino Transferase (ALT), Alkaline Phosphatase (ALP), C-Reactive Protein (CRP) were evaluated and histological examination of organs were done.

Results: After statistical analysis, it was found that the decrease in TLC, RBC, Hb, HCT, and LDH in Wistar rats after RNB intervention in Group A as compared to that of before $R N B$ intervention, was found to be statistically significant $(\mathrm{P}=0.001, \mathrm{P}=0.002, \mathrm{P}=0.001, \mathrm{P}=0.039$, and $\mathrm{P}=0.008)$. On the other hand, an increase was observed in MCV, Urea, Creatinine and ALT values in the Wistar rats after $R N B$ intervention in Group ' $A$ ' as compared to that of before $R N B$ intervention and this increase in values was statistically significant $(\mathrm{P}=0.007, \mathrm{P}=0.001, \mathrm{P}<0.001$ and $\mathrm{P}=0.038)$. After $R N B$ intervention in Group B, the increase in $\mathrm{MCH}$, fibrinogen concentration, and monocytes percentage, was found to be statistically significant $(\mathrm{P}=0.004, \mathrm{P}=0.033$, and $\mathrm{P}=0.001)$ as well as the decrease in $\mathrm{PT}$ and APTT was statistically significant $(\mathrm{P}=0.007$ and $\mathrm{P}=0.002)$. After comparing the Mean Hematobiochemical and coagulation test parameters in the rats of Group A and Group B, after RNB intervention, it was observed that the concentration of Urea, Creatinine, APTT, and D-dimer were less in Group B as compared to that of Group A and this difference was statistically significant $(\mathrm{P}<0.001, \mathrm{P}<0.001, \mathrm{P}<0.001$ and $\mathrm{P}=0.022)$. Histologically the findings in the lungs of group $\mathrm{B}$ were more distortion of lung architecture, most of the alveoli become collapse and make emphysematous changes, more diffuse inflammatory infiltrate within interalveolar septa and around bronchioles as compared to Group A. In the liver of group B rats, the histological findings were mild to moderate distortion of lobular architecture, healthy hepatocytes with more activation of kupffer cells as well as larger and more aggregates of inflammatory cells as compared to group A. Histological findings of kidneys in group A and group B rats were similar to that of control group rats.

Conclusion: The results suggest that the $R N B$ is having an immunomodulatory effect. It might be helpful in the restoration of coagulation factors and can help treat the COVID patients. No harmful effects on the lungs, liver, kidney, and spleen were seen. These findings may act as baseline data for planning further clinical trials in human study subjects to evaluate the effects on various comorbidities.

Keywords: Raj Nirwan Bati, Allovedic, COVID-19, Immunomodulatory, Coagulation, D-dimer

(C) 2022 The Authors. Published by Innovare Academic Sciences Pvt Ltd. This is an open-access article under the CC BY license (https://creativecommons.org/licenses/by/4.0/] DOI: https://dx.doi.org/10.22159/ijpps.2022v14i2.42935. Journal hom epage: https://innovareacademics.in/journals/index.php/ijpps.

\section{INTRODUCTION}

Raj Nirwan Bati (RNB) world's first allovedic medicine that has been formulated at UP University of Medical Sciences, Saifai, Etawah and scientifically proven to be effective in the treatment of COVID-19, can prove to be a milestone in containing this pandemic in India as well as rest of the world. This novel herbomineral formulation has been tested diligently as per contemporary allopathic medical research practices and guidelines. Since it has been tested on all scientific principles of modern medicine (along with the scientific proof of each constituent), it has been termed as allovedic preparation, although it contains established constituents of Ayurveda. The sulfur, probable nano gold and silver particles, Carum copticum, Kharpar (a zinc-containing compound), and pimetine were included for the preparation of $R N B$. These ingredients have immunomodulatory and anti-inflammatory action [1]. Nanoparticles are colloidal carriers. They may be the natural or synthetic origin. Nanoparticles are developed to regulate the release and safeguard drugs from enzymatic or chemical degradation and to improve their therapeutic efficacy [2]. Gold has been considered an inert, noble metal with some therapeutic and medicinal value. Hence, it may be relatively noncytotoxic. Various shapes of these particles can easily be obtained from gold. Its ability for conjugation with peptides and proteins can target the Gold Nano Particles (GNPs) to specific interaction partners. From this point, gold is the most suitable metal for nanotechnology. One of the most affected organs for local and systemic drug delivery is the lung because of its large surface area and close contact with the blood circulation [3]. A comparative evaluation of the biodistribution and the toxic profiles of GNPs and SilverNano Particles (SNPs) showed that their chemical composition played a critical role in their in vivo biodistribution and toxicity. Particularly, GNPs were prominently stored in the liver, whereas SNPs preferentially accumulated more in organs such as the heart, lung, and kidneys [4]. There was evidence that Carum copticum can stimulate beta 2 receptors. Also, it was found that Carum copticum 
inhibits histamine (H1 receptors), thus it has a bronchodilatory and smooth muscle relaxant effect. Substance pimetine (found in Piper nigrum) acts as a mucolytic agent, thereby may reduce the replication of viruses in upper respiratory tract mucosa [1]. Zinc is an excellent antioxidant that prevents the synthesis of oxygen-free radicals that are responsible for oxidative stress [5]. COVID-19, which emerged in Wuhan, Hubei Province, China, is caused by severe acute respiratory syndrome coronavirus 2 (SARS-CoV-2). It is typically spread via respiratory droplets and during close contact. The main clinical manifestation is lung injury. Most of the patients have a favorable prognosis, but some rapidly progress to severe and critical cases with respiratory distress syndrome, coagulation dysfunction, multiple organ failure, etc. Therefore early identification of the severity is very important to the clinical diagnosis of and treatment for COVID-19. Commonly used clinical laboratory coagulation indexes, such as Ddimer (DD), prothrombin time (PT), activated partial thromboplastin time (APTT), thrombin time (TT), and fibrinogen (Fg), could sensitively reflect the clotting state of the body [6]. Abnormal coagulation function, including elevated D-dimer, has been demonstrated to be more common in deceased patients with COVID19 , and increasing odds of in-hospital death were associated with Ddimer greater than $1 \mu \mathrm{g} / \mathrm{ml}[7]$.

This study was conducted to see the effects of Raj NirwanBati (RNB) on hematobiochemical parameters, coagulation tests, and histopathological changes in the lungs, liver, kidneys, and spleen and also to evaluate the immunomodulatory activity of RNB in Wistar rats.

\section{MATERIALS AND METHODS}

In this study, a total of 24 adult albino Wistar rats (of bodyweight 200-250 g) of either sex were used to study the effects of RNB on haematobiochemical parameters, coagulation tests, and histology of organs. Rats were procured from the Animal Husbandry department of UP University of Medical Sciences (UPUMS), Saifai, Etawah \{Committee for the Purpose of Control and Supervision of Experiments on Animals-(CPCSEA) registration no.$1087 / \mathrm{GO} / \mathrm{ReBi} / \mathrm{S} / \mathrm{ReBi} / \mathrm{l} / \mathrm{CPCSEA}\}$ and the experiment were started after taking the approval from Institutional Animal Ethics Committee (IAEC protocol approval no.-IAEC/02/AH/2019-20 dated $07 / 03 / 2020$ ) of UPUMS. An hour before experimentation, animals were brought and kept in the experimental room. Rats were housed under normal conditions and received a standard water and food ad libitum. The rats were weighed, marked, and divided into 3 groups each consisting of 8 animals and the $R N B$ was administered as per the following table (table 1). The blood samples were collected from the jugular vein after giving light anesthesia with Ketamine injection IM. The organ samples (Lungs, Liver, Kidneys, and Spleen) were collected after euthanizing the rats by administration of Ketamine anesthesia overdose IP [8] after the $14^{\text {th }} \mathrm{d}$ of drug administration.

Table 1: Collection of blood samples

\begin{tabular}{|c|c|c|c|c|}
\hline \multirow[t]{2}{*}{ S. No. } & \multirow[t]{2}{*}{ Groups } & \multirow[t]{2}{*}{ No. of rats } & \multirow[t]{2}{*}{$R N B$ dose } & Collection of blood samples \\
\hline & & & & At $0 \mathrm{~d}$ (Before drug) $\quad$ After $14^{\text {th }} \mathrm{d}$ of $R N B$ dose \\
\hline $1-$ & A & 08 & $26 \mathrm{mg} / \mathrm{kg}$ body weight orally & \\
\hline $2-$ & $\mathrm{B}$ & 08 & $260 \mathrm{mg} / \mathrm{kg}$ body weight orally & \\
\hline 3- & Control & 08 & No drug & Only the histology of organs was done. \\
\hline
\end{tabular}

The blood samples taken from the jugular vein were sent to the Central lab within an hour of collection for haematobiochemical examinations. The organ samples were collected in $10 \%$ formalin solution and were sent to the Pathology Department for histopathology. The individual organ index (spleen index) [9] was calculated as follows:-

$$
\text { Spleenindex }=\text { Spleenweight }(\mathrm{g}) / \text { Bodyweight }(\mathrm{g}) \times 100 \%
$$

\section{Test drug}

Raj Nirwan Bati (RNB) was used for testing in this study. It has been formulated at UP University of Medical Sciences, Saifai, Etawah. The main constituents of this novel allovedic preparation are highly purified and processed mercury, processed sulfur, highly purified and almost like nanoparticles of gold and silver, arsenic trioxide (hartala bhasma), black pepper (Piper nigrum), nag daman (Artemisia nilagirica), celery (Carum copticum), Kharpar (A zinccontaining compound), Murdasing, Mukta pishti, and Nirvishi. The quality standards of the ingredients and quality assurance are following the Ayurvedic Pharmacopoeia of India (API) and "General guidelines for Drug Development of Ayurvedic formulations" issued by the Central Council For Research in Ayurvedic Sciences, Ministry of Ayush, Govt. of India, New Delhi, in 2018 [1].

\section{AED (Animal equivalent dose) calculation based on body} surface area

Dose of $R N B$ in adult humans (60 kg bodyweight) $=125 \mathrm{mg}$ per dosage 2 times a day with $5 \mathrm{ml}$ of natural honey [10]

$$
\begin{aligned}
\text { AnimalEquivalentDose(AED) }\left(\frac{\mathrm{mg}}{\mathrm{kg}}\right) & \\
= & \text { HumanDose }\left(\frac{\mathrm{mg}}{\mathrm{kg}}\right) \times \text { Conversionfactor }
\end{aligned}
$$

AED (for rats) $=26 \mathrm{mg} / \mathrm{kg}$ body weight orally with $0.5 \mathrm{ml}$ honey per day X $14 \mathrm{~d}$

After crushing the $R N B$ tablets, it was weighed as per the bodyweight of different rats by using milligram digital balance, and a suspension of $R N B$ was prepared with honey for administration in rats [11].

\section{Hematological studies}

Blood samples in Ethylenediaminetetraacetic acid (EDTA) vials were used to assay hematological parameters. Hematological investigations for the parameters White Blood Cells (WBC) $\left\{\mathrm{X} 10^{3} / \mu \mathrm{l}\right\}$ Red Blood Cells (RBC) $\left.\{\mathrm{X} 106 / \mu \mathrm{l}\}\right)$, Hemoglobin (Hbin g/dl), Hematocrit (HCT in \%), Mean Corpuscular Volume (MCV in fl), Mean Corpuscular Hemoglobin (MCH in pg\}), Mean Corpuscular Hemoglobin Concentration (MCHCin g/dl) were done by using 3Part Automated Hematology Analyzer (fig. 1A) [12].

For determining the number of platelets (PLT) $\left\{\mathrm{X} 10^{3} / \mu \mathrm{l}\right\}$ ) and the Differential Leucocyte Count (DLC) i.e. the percentage of Neutrophils, Lymphocytes, Eosinophils, Monocytes and Basophils, the blood smear was made and stained with May-GrunwaldGiemsa (MGG) stain. Finally, the Neutrophils, Lymphocytes, Eosinophils, Monocytes, and Basophils were counted under a light microscope [13]. Coagulation tests viz. Prothrombin time (PT in s), Activated partial thromboplastin time (APTT in s), Fibrinogen $\{\mathrm{mg} / \mathrm{dl}\}$ and D-dimer $\{\mu \mathrm{g} / \mathrm{ml}\}$ were also done by using Stago machine (fig. 1C) [6]. Neutrophil adhesion tests for the blood samples were done to investigate the immunomodulatory activity of RNB.

Determination of neutrophil adhesion:-After the initial counts, the blood samples were incubated with $80 \mathrm{mg} / \mathrm{ml}$ of nylon fibers for 15 min at $37{ }^{\circ} \mathrm{C}$. The incubated blood samples were again analyzed for TLC and DLC. The product of TLC and \% neutrophil were given as the neutrophil index (NI) of the blood sample. Percent neutrophil adhesion was calculated as follows:

$$
\mathrm{NA}=(\mathrm{NIu}-\mathrm{NIt}) \times 100 / \mathrm{NIu}
$$

Where NA, Neutrophil Adhesion; NIu, neutrophil index of untreated blood sample; NIt, neutrophil index of treated blood sample

$$
\mathrm{NI}=\mathrm{TLC} \times \% \text { neutrophil [14] }
$$




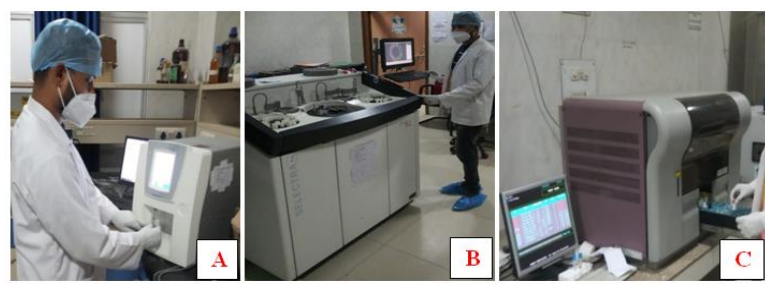

Fig. 1: (A) Medonic three hematology analyzer; (B) Selectra machine for biochemical analysis; (C) Stago machine for coagulation tests

\section{Biochemical studies}

After collecting serum, the serum biochemical parameters-serum Lactate dehydrogenase (LDH in iu/l), Urea $(\mathrm{mg} / \mathrm{dl})$, Creatinine (mg/dl), Aspartate Amino Transferase (AST in iu/l), Alanine Amino Transferase (ALT in iu/l), Alkaline Phosphatase (ALP in iu/l) were determined by using Fully Auto Biochemistry Analyzer Selectra Pro $\mathrm{XL}$ (fig. 1B) [13]. C-Reactive Protein (CRP) was evaluated qualitatively by CARD test.

\section{Histological studies}

The fixed tissues of rats were dehydrated with progressively increasing concentrations of ethanol. The tissues were passed through xylene solution to clear the ethanol and facilitate molten paraffin wax infiltration $\left(55^{\circ} \mathrm{C}\right)$. After that, they were embedded in a wax block. Paraffin sections of 3-4 $\mu$ m thickness were cut with the rotary microtome and placed on cleaned glass slides. Finally, the sections were stained with hematoxylin and eosin. The stained slides were examined using a light microscope, where the photomicrographs of the tissue samples were recorded [13].

\section{Statistical analysis}

For statistical analysis, data were analyzed by Student's paired t-test and one-way Analysis of Variance (ANOVA) using Statistical Package of Social Sciences (SPSS) software version 25, IBM, the USA for windows. The results were expressed as mean \pm standard deviation (SD). Statistical significance was accessed at a $95 \%$ confidence interval and $\mathrm{P}<0.05$ was considered as statistically significant [12].

Table 2: Association of mean haematobiochemical parameters and neutrophil adhesion percentage before and after $R N B$ intervention in both group $A$ and group $B$

\begin{tabular}{|c|c|c|c|c|c|c|c|c|c|c|}
\hline \multirow{2}{*}{$\begin{array}{l}\text { S. } \\
\text { No. }\end{array}$} & \multirow{2}{*}{\multicolumn{2}{|c|}{$\begin{array}{l}\text { Haematobiochemical } \\
\text { parameters (with } \\
\text { Normal range }[15,16] \text { ) }\end{array}$}} & \multirow{2}{*}{$\begin{array}{l}\text { Group A } \\
\text { mean } \pm \text { SD }\end{array}$} & \multirow{3}{*}{$\begin{array}{l}\text { Mean } \\
\text { difference } \pm \\
\text { SD }\end{array}$} & \multirow{3}{*}{$\begin{array}{l}\begin{array}{l}95 \% \\
\text { Confidence } \\
\text { interval (CI) of } \\
\text { the difference }\end{array} \\
3.6-8.5\end{array}$} & \multirow{3}{*}{$\begin{array}{l}\text { Student } \\
\text { pair t- } \\
\text { test-P- } \\
\text { value } \\
0.001^{*}\end{array}$} & \multicolumn{4}{|l|}{ Group B } \\
\hline & & & & & & & mean $\pm S D$ & $\begin{array}{l}\text { Mean } \\
\text { Difference } \pm S\end{array}$ & $\begin{array}{l}95 \% \\
\text { Confidence }\end{array}$ & $\begin{array}{l}\text { Student } \\
\text { pair t- }\end{array}$ \\
\hline 1. & $\begin{array}{l}\text { TLC }[5-13(X \\
\left.\left.10^{3} / \mu \mathrm{l}\right)\right]\end{array}$ & $\begin{array}{l}\text { Before } \\
\text { After }\end{array}$ & $\begin{array}{l}11.43 \pm 2.96 \\
5.34 \pm 1.95\end{array}$ & & & & $\begin{array}{l}5.75 \pm 1.50 \\
6.89 \pm 1.93\end{array}$ & $-1.14 \pm 2.88$ & -3.5 to 1.3 & 0.301 \\
\hline 2. & $\begin{array}{l}\text { RBC }[6-10 \\
\left.\left(\mathrm{X} 10^{6} / \mu \mathrm{l}\right)\right]\end{array}$ & $\begin{array}{l}\text { Before } \\
\text { After }\end{array}$ & $\begin{array}{l}7.66 \pm 0.42 \\
6.84 \pm 0.35\end{array}$ & $0.82 \pm 0.50$ & $0.4-1.2$ & $0.002^{*}$ & $\begin{array}{l}6.84 \pm 0.76 \\
6.69 \pm 0.54\end{array}$ & $0.15 \pm 0.68$ & -0.4 to 0.7 & 0.563 \\
\hline 3. & $\begin{array}{l}\mathrm{Hb}[11-17 \\
(\mathrm{g} / \mathrm{dl})]\end{array}$ & $\begin{array}{l}\text { Before } \\
\text { After }\end{array}$ & $\begin{array}{l}15.05 \pm 0.91 \\
13.40 \pm 0.72\end{array}$ & $1.65 \pm 0.91$ & $0.9-2.4$ & $0.001^{*}$ & $\begin{array}{l}13.08 \pm 1.43 \\
13.34 \pm 0.91\end{array}$ & $-0.26 \pm 1.37$ & -1.4 to 0.9 & 0.605 \\
\hline 4. & $\begin{array}{l}\text { HCT }[38.5-52 \\
(\%)]\end{array}$ & $\begin{array}{l}\text { Before } \\
\text { After }\end{array}$ & $\begin{array}{l}38.70 \pm 2.36 \\
36.05 \pm 2.68\end{array}$ & $2.65 \pm 3.0$ & $0.2-5.1$ & $0.039^{*}$ & $\begin{array}{l}36.7 \pm 2.60 \\
36.64 \pm 3.31\end{array}$ & $0.06 \pm 4.02$ & -3.3 to 3.4 & 0.966 \\
\hline 5. & $\begin{array}{l}\text { MCV [46.3- } \\
57(\mathrm{fl})]\end{array}$ & $\begin{array}{l}\text { Before } \\
\text { After }\end{array}$ & $\begin{array}{l}50.50 \pm 1.99 \\
52.66 \pm 1.76\end{array}$ & $-2.16 \pm 1.64$ & -3.5 to -0.8 & $0.007^{*}$ & $\begin{array}{l}53.95 \pm 3.48 \\
54.75 \pm 2.35\end{array}$ & $-0.80 \pm 3.26$ & -3.5 to 1.9 & 0.510 \\
\hline 6. & $\begin{array}{l}\text { MCH [16.3- } \\
20.3(\mathrm{pg})]\end{array}$ & $\begin{array}{l}\text { Before } \\
\text { After }\end{array}$ & $\begin{array}{l}19.68 \pm 1.49 \\
19.59 \pm 0.29\end{array}$ & $0.09 \pm 1.26$ & 0.9 to 1.1 & 0.850 & $\begin{array}{l}19.15 \pm 0.44 \\
19.95 \pm 0.59\end{array}$ & $-0.80 \pm 0.54$ & -1.25 to -0.3 & $0.004^{*}$ \\
\hline 7. & $\begin{array}{l}\text { МСHC [31.9- } \\
38.5(\mathrm{~g} / \mathrm{dl})]\end{array}$ & $\begin{array}{l}\text { Before } \\
\text { After }\end{array}$ & $\begin{array}{l}38.96 \pm 1.78 \\
37.24 \pm 0.95\end{array}$ & $1.73 \pm 2.17$ & -0.09 to 3.5 & 0.059 & $\begin{array}{l}35.61 \pm 2.31 \\
36.49 \pm 1.06\end{array}$ & $-0.88 \pm 2.66$ & -3.1 to 1.3 & 0.383 \\
\hline 8. & $\begin{array}{l}\text { PLT }[574- \\
1253(X \\
\left.\left.10^{3} / \mu \mathrm{l}\right)\right]\end{array}$ & $\begin{array}{l}\text { Before } \\
\text { After }\end{array}$ & $\begin{array}{l}490 \pm 83.67 \\
310.13 \pm 296.17\end{array}$ & $\begin{array}{l}179.88 \pm 331 \\
75\end{array}$ & -97.4 to 457.2 & 0.169 & $\begin{array}{l}291.75 \pm 120.31 \\
440.5 \pm 183.92\end{array}$ & $\begin{array}{l}-148 \\
75 \pm 253.51\end{array}$ & -360.7 to 63.2 & 0.141 \\
\hline 9. & $\begin{array}{l}\text { Neutrophils } \\
{[9-49(\%)]}\end{array}$ & $\begin{array}{l}\text { Before } \\
\text { After }\end{array}$ & $\begin{array}{l}22.38 \pm 7.07 \\
16.38 \pm 6.52\end{array}$ & $6.0 \pm 7.8$ & -0.5 to 12.50 & 0.066 & $\begin{array}{l}15.25 \pm 3.85 \\
18.25 \pm 3.85\end{array}$ & $-3.0 \pm 5.93$ & -8.0 to 2.0 & 0.195 \\
\hline 10. & $\begin{array}{l}\text { Lymphocytes } \\
{[45-88(\%)]}\end{array}$ & $\begin{array}{l}\text { Before } \\
\text { After }\end{array}$ & $\begin{array}{l}71.13 \pm 4.64 \\
76.38 \pm 8.73\end{array}$ & $-5.25 \pm 8.5$ & -12.3 to 1.8 & 0.123 & $\begin{array}{l}79 \pm 7.41 \\
73.88 \pm 4.39\end{array}$ & $5.13 \pm 10.74$ & -3.9 to 14.1 & 0.219 \\
\hline 11. & $\begin{array}{l}\text { Eosinophils } \\
{[0.3-5(\%)]}\end{array}$ & $\begin{array}{l}\text { Before } \\
\text { After }\end{array}$ & $\begin{array}{l}3.88 \pm 1.13 \\
3.50 \pm 1.60\end{array}$ & $0.38 \pm 2.0$ & -1.3 to 2.0 & 0.612 & $\begin{array}{l}3.25 \pm 0.89 \\
3.88 \pm 1.25\end{array}$ & $-0.63 \pm 1.60$ & -1.9 to 0.7 & 0.305 \\
\hline 12. & $\begin{array}{l}\text { Monocytes [1- } \\
49(\%)]\end{array}$ & $\begin{array}{l}\text { Before } \\
\text { After }\end{array}$ & $\begin{array}{l}2.63 \pm 2.00 \\
3.50 \pm 1.60\end{array}$ & $-0.88 \pm 1.64$ & -2.25 to 0.50 & 0.175 & $\begin{array}{l}2.5 \pm 1.07 \\
4.0 \pm 1.20\end{array}$ & $-1.5 \pm 1.6$ & -2.8 to -0.2 & $0.033^{*}$ \\
\hline 13. & $\begin{array}{l}\text { LDH [256- } \\
1965(\mathrm{iu} / \mathrm{l})]\end{array}$ & $\begin{array}{l}\text { Before } \\
\text { After }\end{array}$ & $\begin{array}{l}3624.50 \pm 1704 . \\
62 \\
1770 \pm 700.39\end{array}$ & $\begin{array}{l}1854.5 \pm 141 \\
7.1\end{array}$ & 669.8 to 3039.2 & $0.008^{*}$ & $\begin{array}{l}568.25 \pm 148.27 \\
2469.38 \pm 2757.82\end{array}$ & $\begin{array}{l}-1901 \\
13 \pm 0.23\end{array}$ & 0.01 to 0.4 & 0.091 \\
\hline 14. & $\begin{array}{l}\text { Urea [10.7- } \\
25(\mathrm{mg} / \mathrm{dl})]\end{array}$ & $\begin{array}{l}\text { Before } \\
\text { After }\end{array}$ & $\begin{array}{l}76.80 \pm 8.63 \\
212.11 \pm 59.04\end{array}$ & $-135.3 \pm 65.6$ & -190.2 to -80.5 & $0.001^{*}$ & $\begin{array}{l}72.38 \pm 32.87 \\
71.81 \pm 6.86\end{array}$ & $0.56 \pm 33.44$ & -27.4 to 28.5 & 0.963 \\
\hline 15. & $\begin{array}{l}\text { Creatinine } \\
{[0.3-0.6} \\
(\mathrm{mg} / \mathrm{dl})]\end{array}$ & $\begin{array}{l}\text { Before } \\
\text { After }\end{array}$ & $\begin{array}{l}0.45 \pm 0.09 \\
1.30 \pm 0.32\end{array}$ & $-0.86 \pm 0.28$ & -1.1 to- 0.6 & $0.000^{*}$ & $\begin{array}{l}0.95 \pm 0.23 \\
0.77 \pm 0.09\end{array}$ & $0.18 \pm 0.23$ & 0.01 to 0.4 & 0.059 \\
\hline 16. & $\begin{array}{l}\text { AST [63- } \\
222(\mathrm{iu} / \mathrm{l})]\end{array}$ & Before & $228.75 \pm 56.51$ & $43.75 \pm 90.82$ & -32.2 to 119.7 & 0.215 & $275.75 \pm 447.15$ & $43.88 \pm 592.51$ & $\begin{array}{l}-451.5 \text { to } \\
539.2\end{array}$ & 0.840 \\
\hline 17. & $\begin{array}{l}\text { ALT [14-64 } \\
(\mathrm{iu} / \mathrm{l})]\end{array}$ & $\begin{array}{l}\text { After } \\
\text { Before }\end{array}$ & $\begin{array}{l}185.0 \pm 74.45 \\
64.0 \pm 26.88\end{array}$ & $-31.0 \pm 34.34$ & -59.7 to -2.3 & $0.038^{*}$ & $\begin{array}{l}231.88 \pm 297.90 \\
115.0 \pm 48.12\end{array}$ & $-60.0 \pm 342.64$ & $\begin{array}{l}-346.5 \text { to } \\
226.5\end{array}$ & 0.636 \\
\hline 18. & $\begin{array}{l}\text { ALP [18- } \\
131(\mathrm{iu} / \mathrm{l})]\end{array}$ & $\begin{array}{l}\text { After } \\
\text { Before }\end{array}$ & $\begin{array}{l}95.0 \pm 27.23 \\
434.0 \pm 142.76\end{array}$ & $1.0 \pm 131.16$ & $\begin{array}{l}-108.65 \text { to } \\
110.65\end{array}$ & 0.983 & $\begin{array}{l}175 \pm 348.01 \\
310.13 \pm 139.98\end{array}$ & $-65.5 \pm 193.24$ & $\begin{array}{l}-227.1 \text { to } \\
96.1\end{array}$ & 0.370 \\
\hline 19. & $\begin{array}{l}\text { Neutrophil } \\
\text { Adhesion } \\
\text { percentage (\%) }\end{array}$ & $\begin{array}{l}\text { After } \\
\text { Before } \\
\text { After }\end{array}$ & $\begin{array}{l}433.0 \pm 35.90 \\
40.17 \pm 30.64 \\
47.19 \pm 13.94\end{array}$ & $-7.01 \pm 34.94$ & -36.2 to 22.2 & 0.588 & $\begin{array}{l}375.63 \pm 143.51 \\
47.29 \pm 32.01 \\
46.39 \pm 13.42\end{array}$ & $-0.91 \pm 36$ & -29.2 to 31.0 & .945 \\
\hline
\end{tabular}

All values are expressed as mean \pm SD. Starmark $(*)$ shows the significant association between means of the respective group when compared with control (before $R N B$ administration) at a $95 \%$ confidence in terval (when $\mathrm{P}<0.05$ ). 


\section{RESULTS}

\section{Haematobiochemical studies}

After statistical analysis (table 2, 3), it was found that the decrease in TLC, RBC, Hb, HCT, and LDH in wistar rats after RNB intervention in Group A i.e. after administration of $R N B @ 26 \mathrm{mg} / \mathrm{kg}$ body weight in rats for a period of $14 \mathrm{~d}$ as compared to that of before $R N B$ intervention, was found to be statistically significant $(\mathrm{P}=0.001$, $\mathrm{P}=0.002, \mathrm{P}=0.001, \mathrm{P}=0.039$, and $\mathrm{P}=0.008$ ). On the other hand, an increase was observed in MCV, Urea, Creatinine and ALT values in the wistar rats after $R N B$ intervention in Group 'A' as compared to that of before $R N B$ intervention and this increase in values was statistically significant $(\mathrm{P}=0.007, \mathrm{P}=0.001, \mathrm{P}<0.001$ and $\mathrm{P}=0.038)$. The differences in the values of $\mathrm{MCH}, \mathrm{MCHC}$, Platelet, neutrophil, lymphocyte, eosinophil, monocyte, AST, ALP, PT, APTT, Fibrinogen, $\mathrm{D}$-dimer and neutrophil adhesion percentage were not statistically significant $(\mathrm{P}=0.850, \mathrm{P}=0.059, \mathrm{P}=0.169, \mathrm{P}=0.066, \mathrm{P}=0.123, \mathrm{P}=0.612$, $\mathrm{P}=0.175, \mathrm{P}=0.215, \mathrm{P}=0.983, \mathrm{P}=0.739, \mathrm{P}=0.063, \mathrm{P}=0.119, \mathrm{P}=0.166$ and $\mathrm{P}=0.588$ ).

In the wistar rats after $R N B$ intervention in Group B i.e. after administration of $R N B @ 260 \mathrm{mg} / \mathrm{kg}$ body weight in rats for a period of $14 \mathrm{~d}$, the increase in $\mathrm{MCH}$, fibrinogen concentration and monocytes percentage, was found to be statistically significant $(\mathrm{P}=0.004, \mathrm{P}=0.033$ and $\mathrm{P}=0.001$ ) as well as the decrease in PT and APTT was statistically significant $(\mathrm{P}=0.007$ and $\mathrm{P}=0.002)$. The differences in the values of TLC, RBCs, Hb, HCT, MCV, MCHC, PLT, neutrophil, lymphocyte, eosinophil, LDH, urea, creatinine, AST, ALT, ALP, D-dimer and neutrophil adhesion percentage were not statistically significant $(\mathrm{P}=0.301, \mathrm{P}=0.563$, $\mathrm{P}=0.605, \quad \mathrm{P}=0.966, \quad \mathrm{P}=0.510, \quad \mathrm{P}=0.383, \mathrm{P}=0.141, \mathrm{P}=0.195, \mathrm{P}=0.219$, $\mathrm{P}=0.305, \mathrm{P}=0.091, \mathrm{P}=0.963, \mathrm{P}=0.059, \mathrm{P}=0.840, \mathrm{P}=0.636, \mathrm{P}=0.370$, $\mathrm{P}=0.210$ and $\mathrm{P}=0.945)$.

After comparing the Mean Haematobiochemical parameters, neutrophil adhesion percentage, and coagulation test parameters in the rats of Group A and Group B, after RNB intervention (table 4 and 5), it was observed that the concentration of Urea, Creatinine, APTT, and D-dimer were less in Group B as compared to that of Group A and this difference was statistically significant $(\mathrm{P}<0.001, \mathrm{P}<0.001$, $\mathrm{P}<0.001$ and $\mathrm{P}=0.022$ ) and the differences in the values of TLC, RBC, $\mathrm{Hb}, \mathrm{HCT}, \mathrm{MCV}, \mathrm{MCH}, \mathrm{MCHC}$, PLT, neutrophil, lymphocyte, eosinophil, monocyte, LDH, AST, ALT, ALP, neutrophil adhesion percentage, PT and fibrinogen were found to be statistically non-significant $(\mathrm{P}=0.133, \mathrm{P}=0.532, \mathrm{P}=0.881, \mathrm{P}=0.702, \mathrm{P}=0.064, \mathrm{P}=0.14, \mathrm{P}=0.159$, $\mathrm{P}=0.308, \mathrm{P}=0.495, \mathrm{P}=0.481, \mathrm{P}=0.610, \mathrm{P}=0.491, \mathrm{P}=0.498, \mathrm{P}=0.672$, $\mathrm{P}=0.527, \mathrm{P}=0.291, \mathrm{P}=0.908, \mathrm{P}=0.95$ and $\mathrm{P}=0.30$ ).

It was also seen that the difference in spleen index in Group A and Group B i.e. after $R N B$ administration as compared to the control group, was statistically non-significant $(\mathrm{P}=0.946)$ (table 6).

Table 3: Association of mean coagulation test parameters before and after $R N B$ intervention in group 'A' and Group 'B

\begin{tabular}{|c|c|c|c|c|c|c|c|c|c|c|}
\hline \multirow{2}{*}{$\begin{array}{l}\text { S. } \\
\text { No. }\end{array}$} & \multirow{2}{*}{\multicolumn{2}{|c|}{$\begin{array}{l}\text { Coagulation test } \\
\text { (with normal range } \\
{[17] \text { ) }}\end{array}$}} & \multicolumn{4}{|l|}{ Group A } & \multicolumn{4}{|l|}{ Group B } \\
\hline & & & mean \pm SD & $\begin{array}{l}\text { Mean } \\
\text { difference } \pm \\
\text { SD }\end{array}$ & $\begin{array}{l}95 \% \\
\text { confidence } \\
\text { interval (CI) of } \\
\text { the difference }\end{array}$ & $\begin{array}{l}\text { Student } \\
\text { pair t- } \\
\text { test-P- } \\
\text { value } \\
\end{array}$ & mean $\pm S D$ & $\begin{array}{l}\text { Mean } \\
\text { difference } \pm S D\end{array}$ & $\begin{array}{l}95 \% \\
\text { confidence } \\
\text { interval (CI) of } \\
\text { the difference } \\
\end{array}$ & $\begin{array}{l}\text { Student } \\
\text { pair t- } \\
\text { test-P- } \\
\text { value } \\
\end{array}$ \\
\hline \multirow[t]{2}{*}{1.} & \multirow{2}{*}{$\begin{array}{l}\text { PT (24.5- } \\
30.9 \mathrm{~s})\end{array}$} & Before & $24.83 \pm 13.27$ & $1.41 \pm 11.54$ & -8.2 to 11.1 & 0.739 & $27.45 \pm 4.88$ & $4.14 \pm 3.13$ & -1.52 to 6.8 & $0.007^{*}$ \\
\hline & & After & $23.41 \pm 3.61$ & & & & $23.31 \pm 2.58$ & & & \\
\hline \multirow[t]{2}{*}{2.} & \multirow{2}{*}{$\begin{array}{l}\text { APTT (13.0- } \\
19.2 \mathrm{~s})\end{array}$} & Before & $30.65 \pm 4.47$ & $-4.45 \pm 5.69$ & -9.2 to 0.31 & 0.063 & $25.76 \pm 4.40$ & $5.89 \pm 3.59$ & 2.9 to 8.9 & $0.002^{*}$ \\
\hline & & After & $35.10 \pm 8.09$ & & & & $19.88 \pm 2.03$ & & & \\
\hline \multirow[t]{2}{*}{3.} & \multirow{2}{*}{$\begin{array}{l}\text { Fibrinogen } \\
(160-255 \\
\mathrm{mg} / \mathrm{dl})\end{array}$} & Before & $131.75 \pm 65.01$ & $\begin{array}{l}-26.13 \pm 41 \\
59\end{array}$ & -60.9 to 8.65 & 0.119 & $114.38 \pm 47.99$ & $-75.13 \pm 39.73$ & -108.3 to-41.9 & $0.001^{*}$ \\
\hline & & After & $157.88 \pm 70.80$ & & & & $189.50 \pm 43.68$ & & & \\
\hline \multirow[t]{2}{*}{4.} & \multirow{2}{*}{$\begin{array}{l}\text { D-dimer [0- } \\
0.5(\mu \mathrm{g} / \mathrm{dl})]\end{array}$} & Before & $1.36 \pm 1.61$ & \multirow[t]{2}{*}{$-0.30 \pm 0.54$} & \multirow[t]{2}{*}{-0.8 to 0.2} & \multirow[t]{2}{*}{0.166} & $0.40 \pm 0.26$ & $0.12 \pm 0.24$ & -0.1 to 0.3 & 0.210 \\
\hline & & After & $1.65 \pm 1.51$ & & & & $0.28 \pm 0.07$ & & & \\
\hline
\end{tabular}

Starmark $(*)$ shows the significant association between means of the respective group when compared with control (before RNB administration) at a $95 \%$ confidence interval (when $\mathrm{P}<0.05$ ).

Table 4: Association of mean haematobiochemical parameters and neutrophil adhesion percentage after $R N B$ intervention in group A and group B

\begin{tabular}{|c|c|c|c|c|c|c|}
\hline \multirow[t]{2}{*}{ S. No. } & \multirow[t]{2}{*}{ Haematobiochemical parameters } & \multirow[t]{2}{*}{ Normal range $[15,16]$} & \multicolumn{2}{|l|}{ mean \pm SD } & \multirow[t]{2}{*}{ F-Value } & \multirow[t]{2}{*}{ P-Value } \\
\hline & & & Group A & Group B & & \\
\hline 1. & TLC & $5-13\left(\mathrm{X} 10^{3} / \mu \mathrm{l}\right)$ & $5.34 \pm 1.95$ & $6.89 \pm 1.93$ & 0.12 & 0.133 \\
\hline 2. & $\mathrm{RBC}$ & $6-10\left(X 10^{6} / \mu \mathrm{l}\right)$ & $6.84 \pm 0.35$ & $6.69 \pm 0.54$ & 1.54 & 0.532 \\
\hline 3. & $\mathrm{Hb}$ & $11-17(\mathrm{~g} / \mathrm{dl})$ & $13.4 \pm 0.72$ & $13.34 \pm 0.91$ & 1.49 & 0.881 \\
\hline 4. & HCT & $38.5-52(\%)$ & $36.05 \pm 2.68$ & $36.64 \pm 3.31$ & 1.37 & 0.702 \\
\hline 5. & MCV & $46.3-57(\mathrm{fl})$ & $52.66 \pm 1.76$ & $54.75 \pm 2.35$ & 1.25 & 0.064 \\
\hline 6. & $\mathrm{MCH}$ & $16.3-20.3(\mathrm{pg})$ & $19.59 \pm 0.29$ & $19.95 \pm 0.59$ & 2.18 & 0.14 \\
\hline 7. & $\mathrm{MCHC}$ & $31.9-38.5(\mathrm{~g} / \mathrm{dl})$ & $37.24 \pm 0.95$ & $36.49 \pm 1.06$ & 0.03 & 0.159 \\
\hline 8. & PLT & $574-1253\left(\mathrm{X} 10^{3} / \mu \mathrm{l}\right)$ & $310.13 \pm 296.17$ & $440.50 \pm 183.92$ & 13.91 & 0.308 \\
\hline 9. & Neutrophil & $9-49(\%)$ & $16.38 \pm 6.52$ & $18.25 \pm 3.85$ & 1.53 & 0.495 \\
\hline 10. & Lymphocyte & $45-88(\%)$ & $76.38 \pm 8.73$ & $73.88 \pm 4.39$ & 2.54 & 0.481 \\
\hline 11. & Eosinophil & $0.3-5(\%)$ & $3.50 \pm 1.60$ & $3.88 \pm 1.25$ & 0.20 & 0.610 \\
\hline 12. & Monocyte & $1-4(\%)$ & $3.5 \pm 1.6$ & $4.0 \pm 1.20$ & 0.47 & 0.491 \\
\hline 13. & $\mathrm{LDH}$ & $256-1965(\mathrm{iu} / \mathrm{l})$ & $1770 \pm 700.39$ & $2469.38 \pm 2757.82$ & 5.95 & 0.498 \\
\hline 14. & Urea & $10.7-25(\mathrm{mg} / \mathrm{dl})$ & $212.11 \pm 59.04$ & $71.81 \pm 6.86$ & 11.88 & $<0.001^{*}$ \\
\hline 15. & Creatinine & $0.3-0.6(\mathrm{mg} / \mathrm{dl})$ & $1.30 \pm 0.32$ & $0.77 \pm 0.09$ & 2.27 & $<0.001^{*}$ \\
\hline 16. & AST & $63-222(\mathrm{iu} / \mathrm{l})$ & $185 \pm 74.45$ & $231.88 \pm 297.9$ & 3.24 & 0.672 \\
\hline 17. & ALT & $14-64(\mathrm{iu} / \mathrm{l})$ & $95.0 \pm 27.26$ & $175.0 \pm 348.01$ & 4.28 & 0.527 \\
\hline 18. & ALP & $18-131(\mathrm{iu} / \mathrm{l})$ & $433 \pm 35.90$ & $375.63 \pm 143.51$ & 5.2 & 0.291 \\
\hline 19. & Neutrophil adhesion (NA) percentage (\%) & & $47.19 \pm 13.42$ & $46.39 \pm 13.42$ & 0.097 & 0.908 \\
\hline
\end{tabular}

Starmark (*) shows the significant association between means of group 'A' and group 'B' (after RNB administration) at a 95\% confidence interval (when $\mathrm{P}<0.05$ ). 
Table 5: Association of mean coagulation test parameters after $R N B$ intervention in group ' $A$ ' and group 'B'

\begin{tabular}{|c|c|c|c|c|c|c|}
\hline \multirow[t]{2}{*}{ S. No. } & \multirow[t]{2}{*}{ Coagulation test } & \multirow[t]{2}{*}{ Normal range [17] } & \multicolumn{4}{|l|}{ mean \pm SD } \\
\hline & & & Group A & Group B & F-value & P-value \\
\hline 1. & PT & $24.5-30.9 \mathrm{~s}$ & $23.41 \pm 3.61$ & $23.31 \pm 2.58$ & 0.61 & 0.95 \\
\hline 2. & APTT & $13.0-19.2 \mathrm{~s}$ & $35.10 \pm 8.09$ & $19.88 \pm 2.03$ & 9.54 & $<0.001$ * \\
\hline 3. & Fibrinogen & $160-255 \mathrm{mg} / \mathrm{dl}$ & $157.88 \pm 70.80$ & $189.5 \pm 43.68$ & 3.08 & 0.30 \\
\hline 4. & D-dimer & $0-0.5 \mu \mathrm{g} / \mathrm{dl}$ & $1.65 \pm 1.51$ & $0.28 \pm 0.07$ & 5.25 & $0.022^{*}$ \\
\hline
\end{tabular}

Starmark $(*)$ shows the significant association between means of group 'A' and group 'B' (after $R N B$ administration) at a $95 \%$ confidence interval (when $\mathrm{P}<0.05$ ).

Table 6: $R N B$ mediated variations in the spleen index (\%) of wistar rats [Values are expressed as mean \pm SD]

\begin{tabular}{llll}
\hline S. No. & Group & Spleen index (mean \pm SD) & Statistical value* (P-value) \\
\hline 1. & Control & $0.20 \pm 0.04$ & 0.946 \\
2. & A & $0.20 \pm 0.03$ & \\
3. & B & $0.21 \pm 0.04$ & \\
\hline
\end{tabular}

${ }^{*}$ ANOVA test

Table 7: Findings of histopathological studies

\begin{tabular}{|c|c|c|c|c|}
\hline S. No. & Organ & Control group & Group A & Group B \\
\hline 1. & Lungs & $\begin{array}{l}\text { - Normal lung with a spongy structure. } \\
\text { •Normal clear alveoli and thin } \\
\text { interalveolar septa. } \\
\text { •Diffuse inflammatory infiltrates within } \\
\text { the interalveolar septa are absent } \\
\text { whereas minimal inflammatory infiltrates } \\
\text { around the bronchioles are present. } \\
\bullet \text { Occasional vacuolation in the bronchial } \\
\text { wall and no desquamation of lining } \\
\text { epithelium with normal thickening. } \\
\text { •Blood vessels-dilated and very less } \\
\text { thickened. } \\
\bullet \text { Very minimal inflammation and no } \\
\text { collection of lymphoid aggregates in the } \\
\text { interstitium. }\end{array}$ & $\begin{array}{l}\text {-Mild loss of lung parenchyma. } \\
\text {-Inter alveolar septa-slightly } \\
\text { thickened and collapse of some } \\
\text { alveoli compared to control group. } \\
\text { •Mild mixed inflammatory infiltrates } \\
\text { within the interalveolar septa. } \\
\text { •Bronchial walls show some } \\
\text { vacuolation, desquamation of lining } \\
\text { epithelium with a focal increase in } \\
\text { thickness compared to the control } \\
\text { group. } \\
\text { •Mildly dilated and congested blood } \\
\text { vessels with normal thickening. } \\
\bullet \text { Occasional lymphoid aggregate in } \\
\text { the interstitium. }\end{array}$ & $\begin{array}{l}\bullet \text { More distortion of lung architecture. Most } \\
\text { of the alveoli become collapse and make } \\
\text { emphysematous changes. } \\
\bullet \text { Diffuse inflammatory infiltrates within } \\
\text { interalveolar septa (more thickened) and } \\
\text { around bronchioles are more as compared } \\
\text { to Group A. •Vacuolation-more in these } \\
\text { bronchial walls and some desquamation of } \\
\text { lining epithelium with mild thickening. } \\
\bullet \text { More dilation and congestion of blood } \\
\text { vessels and media revealed splitting and } \\
\text { disorganization of its layers compared to } \\
\text { group A. } \\
\bullet \text { A scattered collection of lymphoid } \\
\text { aggregates in the interstitium is more } \\
\text { compared to group A. }\end{array}$ \\
\hline 2. & Liver & $\begin{array}{l}\text { - main tained lobular architecture. } \\
\text {-Hepatocytes are looking healthy with } \\
\text { very minimal activation of Kupffer cells. } \\
\text { •Hepatocytes show the occasional } \\
\text { aggregate of inflammatory cells. } \\
\text { •Portal tracts are normal and show } \\
\text { very minimal mixed inflammatory cell } \\
\text { infiltrate around them. } \\
\text { •Sinusoids-normal and filled with } \\
\text { blood. }\end{array}$ & $\begin{array}{l}\text {-Maintained lobular architecture with } \\
\text { normal and healthy hepatocytes. Some } \\
\text { binucleated cells refer to regeneration. } \\
\text {-More activation of kupffer cells and } \\
\text { more aggregate of inflammatory cells } \\
\text { as compared to the control group. } \\
\text {-Portal tracts-normal and show more } \\
\text { mixed inflammatory cell infiltrate } \\
\text { around it compared to the control } \\
\text { group. } \\
\text {-Sinusoids-unremarkable and filled } \\
\text { with blood. }\end{array}$ & $\begin{array}{l}\text { - Mild to moderate distortion of lobular } \\
\text { architecture. } \\
\text { •Hepatocytes-healthy with more activation } \\
\text { of kupffer cells as well as larger and more } \\
\text { aggregates of inflammatory cells as } \\
\text { compared to group A. } \\
\text {-Portal tracts-moderate mixed } \\
\text { inflammatory infiltrate higher than group A. } \\
\text { •Sinusoids-slightly more dilated and filled } \\
\text { with blood. } \\
\text { - Focal steatosis in the form of micro and } \\
\text { macro vesicles is seen. }\end{array}$ \\
\hline 3. & Kidney & $\begin{array}{l}\text { - Normal renal parenchyma with well- } \\
\text { developed corticomedullary } \\
\text { differentiation. } \\
\text { •Glomeruli are healthy and show normal } \\
\text { glomerular tuft. Tubules are maintained. } \\
\text { • Blood vessels-largely unremarkable. } \\
\text { Sections from bilateral adrenal glands } \\
\text { are unremarkable. }\end{array}$ & $\begin{array}{l}\text {-Similar findings as in the control } \\
\text { group. }\end{array}$ & $\begin{array}{l}\text {-Similar findings as in the control group } \\
\text { and the tubules are mildly dilated. }\end{array}$ \\
\hline 4. & Spleen & $\begin{array}{l}\bullet \text { Maintained splenic architecture with } \\
\text { normal lymphoid follicles. } \bullet \text { Sinusoids- } \\
\text { normally present and filled with blood. } \\
\bullet \text { White pulps-homogenously present } \\
\text { and unremarkable. } \\
\bullet \text { Red pulps-unremarkable and show } \\
\text { hemorrhagic areas at places. }\end{array}$ & $\begin{array}{l}\text {-Similar findings as in control group } \\
\text { and } \\
\text { •Few granular leukocytes, along with } \\
\text { large macrophages are present in } \\
\text { between the lymphocytes in } \\
\text { lymphoid follicles. }\end{array}$ & $\begin{array}{l}\text {-Maintained splenic architecture with } \\
\text { variably sized lymphoid follicles. } \\
\text {-Sinusoids-more dilated and filled with } \\
\text { blood as compared to group A. } \\
\text {-White pulps-slightly atrophied. } \\
\text {-Red pulps-mildly expanded and show } \\
\text { hemorrhagic areas at places. } \\
\text {-The presence of granular leukocytes with } \\
\text { a minimal increase of histiocytes is more in } \\
\text { between the lymphocytes in lymphoid } \\
\text { follicles as compared to group A. }\end{array}$ \\
\hline
\end{tabular}

$C R P$, evaluated by qualitative method, was non-reactive in all rat groups, before and after $R N B$ intervention. 


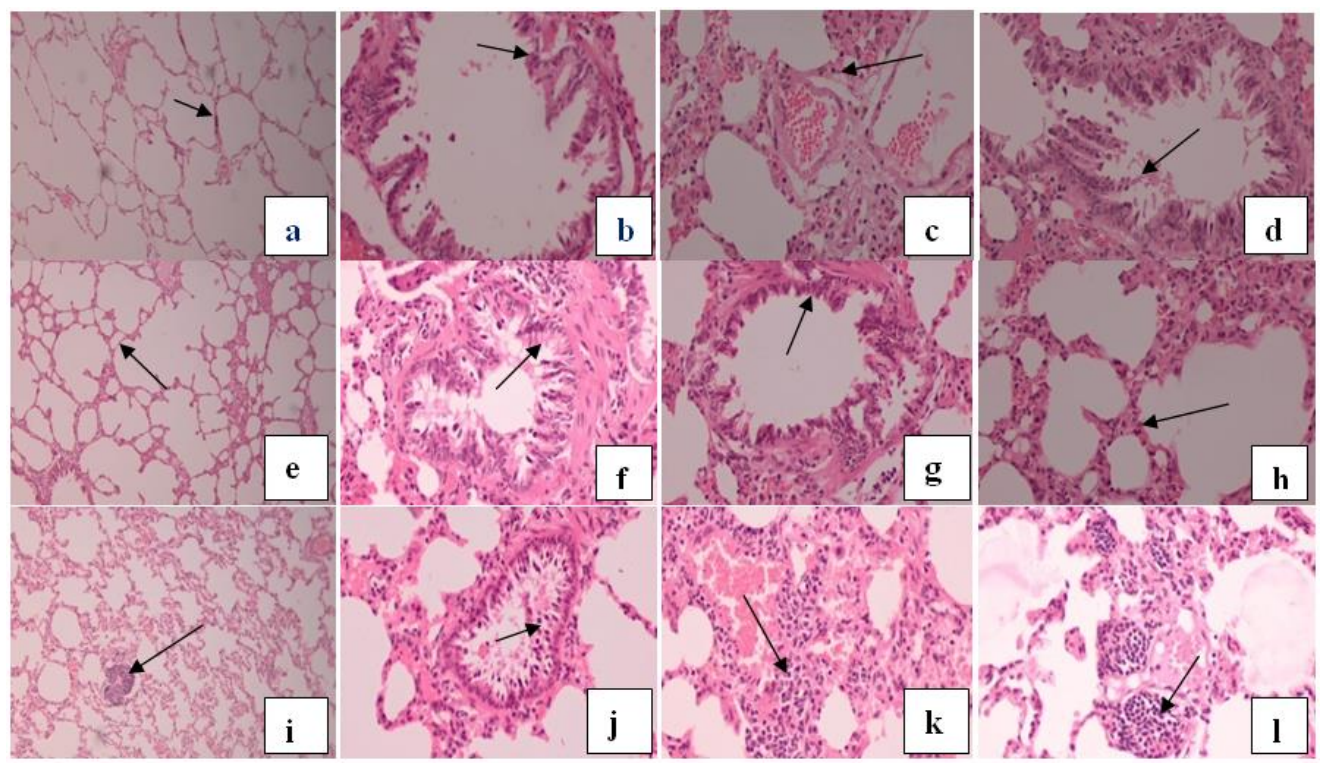

Fig. 1: Photomicrographs of histology of rat Lungs: fig. a, b, $c$ and d-Control group rat lungs $\{(a)$, Normal clear alveoli with thin interalveolar septa,10X, (b)Very minimal inflammatory infiltrate around the bronchiole,40X, (c) Diffuse inflammatory infiltrate within interalveolar septa are absent, 40X, (d) Occasional vacuolation is seen in the bronchial wall and no desquamation of lining e pithelium, 40X $\}$; fig. e, $f, g$ and $h$-Group ' $A$ ' rat lungs $\{(e)$ Interalveolar septa are slightly thickened and collapse of some alveoli compared to control group,10X, (f) Bronchial walls show desquamation, 40X, (g) Bronchial walls show vacuolation of lining epithelium with a focal increase in thickness, $40 \mathrm{X}$, (h) Minimal inflammatory infiltrate around septa, 40X\}; fig. i, j, k and l-Group 'B' rat lungs \{(i) Lungs show more distortion of lung architecture, most alveoli become collapsed and make emphysematous changes with lymphoid aggregate as compared to group ' $A$ ', 10X, (j) Diffuse inflammatory infilterate within interalveolar septa and inflammatory infilterate around the bronchioles are more as compared to group ' $A$ ' and vacuolation is more in the bronchial wall and some desquamation in lining epithelium, 40X, (k) Interalveolar septa are more thickened with more mixed inflammatory infilterate as compared to group A,40X, (1) Scattered lymphoid aggregate in the interstitium are more as compared to group $\mathrm{A}, 40 \mathrm{X}$ \} (as shown by arrows)

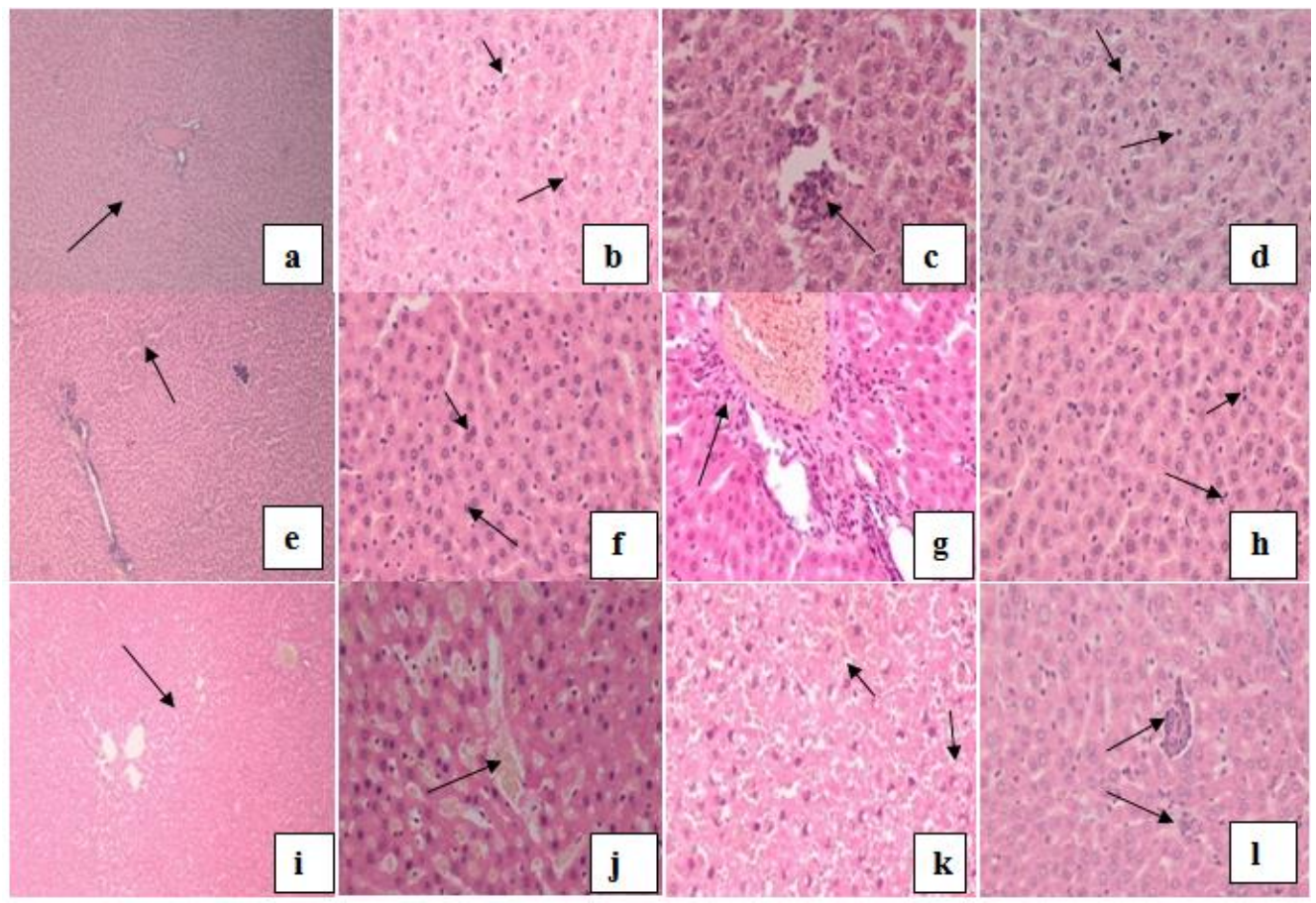

Fig. 2: Photomicrographs of histology of rat Liver: fig. a, b, c and d-Control group rat liver \{(a)Maintained lobular architecture, 10X, (b) Minimal activation of kupffer cells,40X, (c) Occasional aggregate of lymphoid cells, 40X, (d) Normal sinusoids filled with blood and prominence ofkupffer cells, 40X\}; fig. e, f, gand $h$-Group 'A' rat liver $\{(e)$ Liver parenchyma show slightly more aggregates of inflammatory cells as compared to control group,10X, (f) Some binucleate cells refers to regeneration, 40X, (g) Portal tracts are normal and show slightly more mixed inflammatory infiltrate around it, $40 \mathrm{X}$, (h) More activation of kupffer cells as compared to control group, 40X\}; fig. i, $\mathrm{j}$, $\mathrm{k}$ and l-Group 'B' rat liver \{(i) Liver parenchyma showmild to moderate distortion of lobular architecture, 10X, (j) Sinusoids are slightly more dilated and filled with blood, 40X, (k) Focal steatosis in form of micro and macro-vescicles which is not present in the control group and Group ' $\mathrm{A}$ ',40X, (l) Liver parenchyma show larger as well as more aggregates of inflammatory cells as compared to group $\mathrm{A}, 40 \mathrm{X}$ \} (as shown by arrows) 


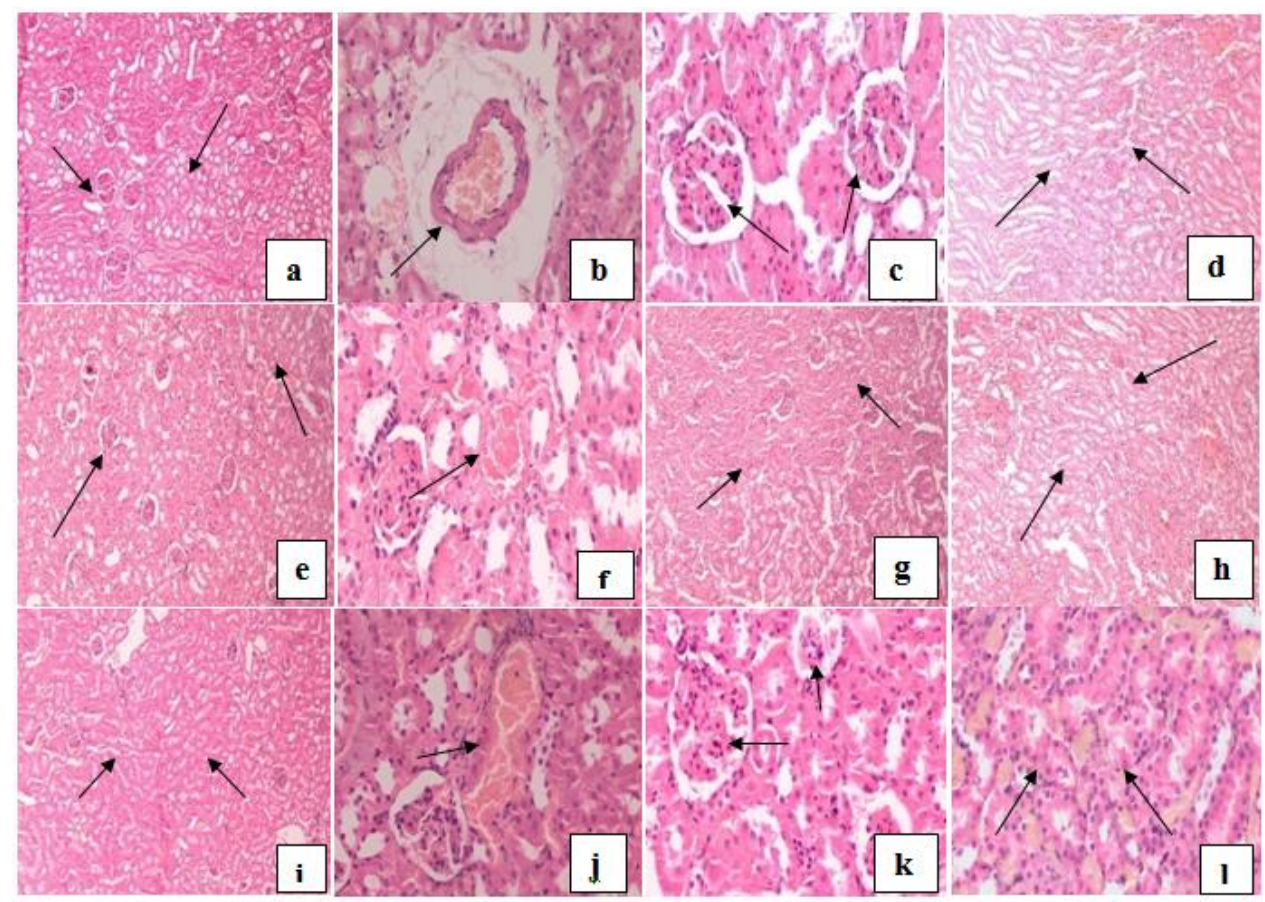

Fig. 3: Photomicrographs of histology of rat kidney: fig. a, b, c and d-Control group rat kidney \{(a) Bilateral kidney show normal renal parenchyma with well-developed corticomedullary differentiation, 10X, (b) Blood vessels unremarkable,40X, (c) Glomeruli healthy and show normal glomerular tuft, 40X, (d) Tubules unremarkable, 40X\}; fig. e, f, g and h-Group ' $A$ ' rat kidney $\{($ e) Bilateral kidney show normal renal parenchyma with well-developed corticomedullary differentiation, $10 X$, (f) Blood vessels unremarkable, 40X, (g) Corticomedullary differentiation unremarkable, $40 \mathrm{X}$, (h) Medulla unremarkable, 40X\}; fig. i, j, k and l-Group 'B' rat kidney \{(i) Bilateral kidney show normal renal parenchyma with well-developed corticomedullary differentiation, 10X, (j) Blood vessels unremarkable, 40X, (k) Healthy glomeruli,40X, (l) Tubules unremarkable, 40X\} (as shown by arrows)

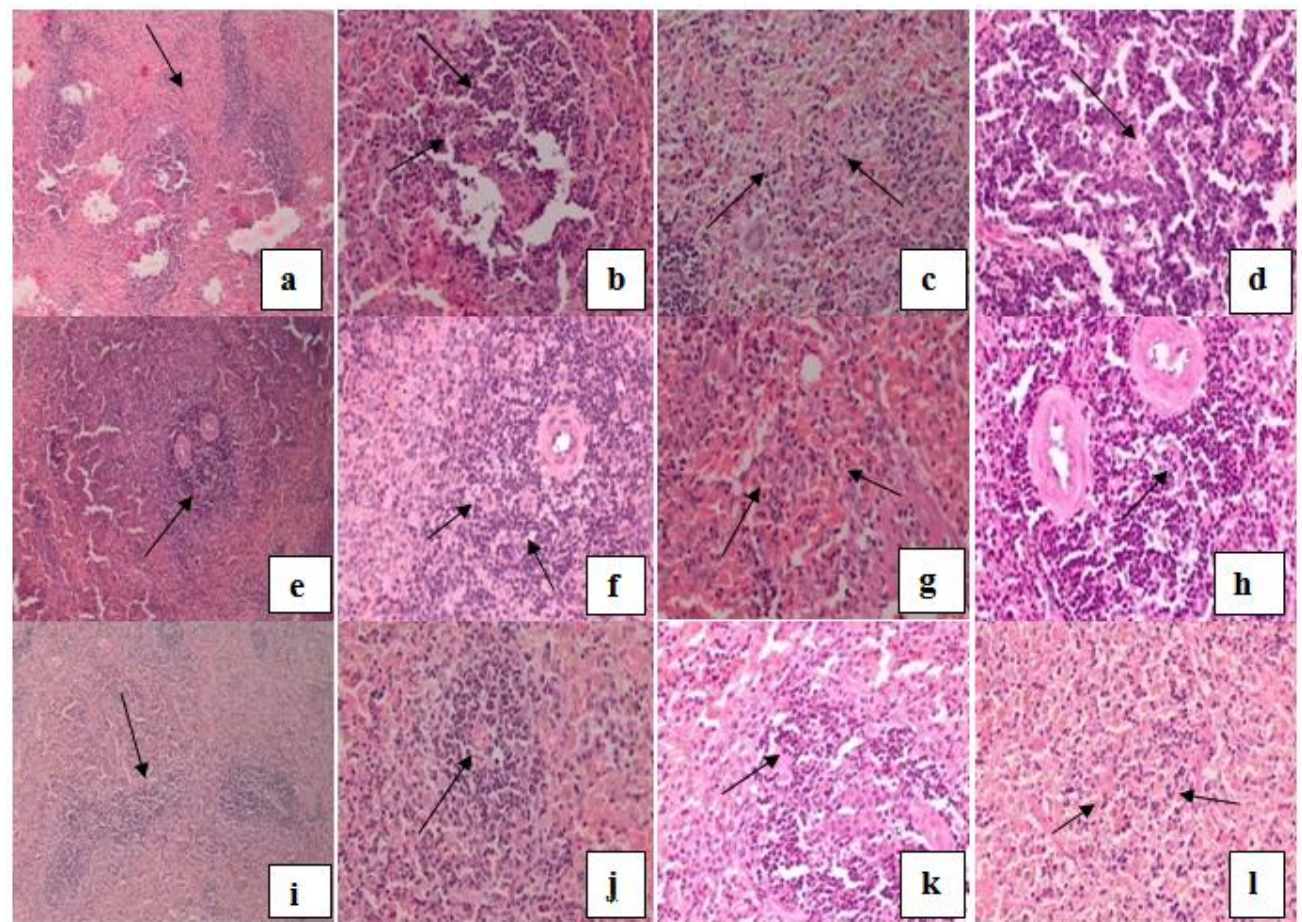

Fig. 4: Photomicrographs of Histology of rat Spleen: fig. a, b, c and d-Control group rat spleen \{(a) Maintained splenic architecture with the normal lymphoid follicle, 10X, (b) White pulp homogenously present and are unremarkable,40X, (c) Red pulp unremarkable and sh ow

hemorrhagic areas at places, $40 \mathrm{X}$, (d) Occasional histiocytes in between the follicles, $40 \mathrm{X}\}$; fig. $\mathrm{e}, \mathrm{f}, \mathrm{g}$ and $\mathrm{h}$-Group ' $\mathrm{A}$ ' rat spleen $\{(\mathrm{e})$ Maintained splenic architecture with normal lymphoid follicle,10X, (f) Histiocytes are slightly more along with few polymorph s, 40X, (g) Red pulp unremarkable and show hemorrhagic areas at places, $40 \mathrm{X}$, (h) Few granular lymphocytes along with macrophages are present in between lymphocytes in lymphoid follicles, 40X\}; fig. $i, j, k$ and l-Group 'B' rat spleen $\{(i)$ and (j) Expansion of red pulp and atrophy of white pulp at10X and 40X respectively, (k) Presence of granular leucocytes with minimal increase of histiocytes as compared to group 'A',40X, (1) Red pulps are mildly expanded and filled with blood, 40X) (as shown by arrows) 


\section{DISCUSSION}

In the present study, significant changes were observed in some haematobiochemical parameters in Group A in which a therapeutic dose of $R N B @ 26 \mathrm{mg} / \mathrm{kg}$ was given but there were no significant changes in these parameters in Group B in which ten times higher dose of $R N B @ 260 \mathrm{mg} / \mathrm{kg}$ was given. So these changes in Group A might be due to incidental and agree with the findings of a previous study [12] in which PurnaCantirotayaCenturam (PCC), a herbometallic formulation of Siddha medicine, consisting of mercury, sulfur, and gold processed with red cotton flower and plantain stem pith juices was used to evaluate its safety and acute and 28-d repeated oral toxicity studies were performed. There was no hematological variation noted between vehicle control and test drug-treated groups. The results of the hematological parameters of this study did not show any worriso me results since all the changes were within the normal expected range for the rat species used in this study. In this study, there were some significant changes observed in urea, creatinine, cholesterol, and albumin parameters between the vehicle control group and low dose $2.5 \mathrm{mg} / \mathrm{kg}$ body weight group, which might be due to incidental. Histopathological findings were also normal in the low dose group and unsubstantiated these chemical alterations. In this study, there were no significant changes in the levels of creatinine, urea, sodium, potassium, and chloride in the sera of the other two groups of rats treated with different doses of $P C C$ and therefore, they were considered non-nephrotoxic. Transaminases (SGOT and SGPT) are good indicators of liver function and biomarkers to predict the possible toxicity of drugs [18]. There were no changes in the SGPT and SGOT levels which reveal that PCC did not affect liver function. There were no significant differences in other biochemical parameters between vehicle control and test groups [12], which is certainly in support of the findings of our study.

In serum, the assessment and determination of liver enzymes such as ALT, AST, ALP, LDH, serum lipid profile, cholesterol, triacylglycerides, and lipoproteins are providing a clear picture of the liver function in an animal. It is also used to evaluate the exact functional status of the liver and to detect liver cellular injury that occurred in the animal. Most of the experimental studies suggested that increased levels of urea, nitrogen, uric acid, and creatinine concentrations in blood reflected the dysfunction of kidneys in animals [19]. These findings are in agreement with the findings of the present study, as in Group B rats in which a higher dose of RNB was administered, the difference in the values of LDH, urea, creatinine, AST, ALT, and ALP, were not statistically significant indicating no adverse effects of RNB on liver and kidneys even after administration of 10 times higher dose.

In our study, it was observed that there was an increase in monocytes percentage in both groups $\mathrm{A}$ and $\mathrm{B}$ and this increase was statistically significant in group B, indicating more number of macrophages and increased phagocytic activity. The macrophages are formed through the differentiation of monocytes, one of the major groups of white blood cells of the immune system. When there is tissue damage or infection, the monocytes leave the bloodstream and enter the affected tissue or organ and undergo a series of changes to become macrophages. These macrophages can modify themselves to form different structures to fight various microbes and invaders. In this way, macrophages provide the first line of defense in protecting the host from infection [20]. Thus it indicates the immunomodulatory effect of $R N B$.

In our study, the difference observed in D-dimer level in group $A$ and $\mathrm{B}$ after $R N B$ intervention as compared to before $R N B$ intervention was not significant, but on comparing the values of D-dimer in between groups A and B after RNB intervention, it was found that the concentration of D-dimer was less (but within normal limit) in Group B as compared to Group A, which was found to be statistically significant and this lowered concentration of D-dimer is due to the effect of $R N B$ administered @260 mg/kg body weight for $14 \mathrm{~d}$. This finding also indicates that RNB might be helpful in the treatment of COVID patients as abnormal coagulation function, including elevated D-dimer, has been demonstrated to be involved in the disease progression of COVID-19[21,22]. In a study, the association between elevated D-dimer levels and the disease severity of COVID-19 were analyzed based on the evidence from the cohort study and metaanalysis. In this study, the level of D-dimer was markedly increased in patients with severe COVID-19, and the meta-analysis further confirmed that odds of severe COVID-19 were associated with Ddimer greater than $0.5 \mu \mathrm{g} / \mathrm{ml}$. In this cohort study, the level of coagulation function parameters, including prothrombin time, fibrinogen, fibrin degradation products, and D-dimer, were found elevated in patients with severe COVID-19. Presumably, the severity of COVID-19 might also be associated with coagulation dysfunction [7]. Moreover, the increase of D-dimer may be an indirect manifestation of inflammatory reaction, as inflammatory cytokines could cause the imbalance of coagulation and fibrinolysis in the alveoli, which may activate the fibrinolysis system, and then increase the level of $\mathrm{D}$-dimer $[23,24]$. D-dimer greater than $1 \mu \mathrm{g} / \mathrm{ml}$ was found a risk factor of poor prognosis for patients with COVID-19 [22]. Therefore, the findings related to D-dimer in our study are in support of the beneficial effects of $R N B$ administration in COVID patients.

A study was conducted for investigating the role of the dynamic changes of D-dimer, PT, APTT, TT, and fibrinogen in predicting the severity and prognosis in patients with COVID-19. The results of this study showed that the levels of D-dimer, PT, and APTT were significantly higher whereas Fibrinogen in deceased cases was significantly lower than those in survival cases, suggesting the dynamic coagulating process in patients with COVID-19 is likely the hypercoagulation state followed by the activation of fibrinolysis. In this study, PT and APTT were prolonged in 23 deceased patients, and the prolongation was more significant in the second and third tests, indicating the patients were in the transition from the high coagulating state into the fibrinolytic state due to the excessive consumption of coagulating factors. Additionally, the study results showed D-dimer, one of the fibrinolytic degradation products, gradually increased throughout the disease, indicating that the patients were possibly in the hyperfibrinolytic state [6]. The results of our study showed that in Group B, PT and APTT were significantly reduced and the fibrinogen was significantly increased after administration of $R N B @ 260 \mathrm{mg} / \mathrm{kg}$ bodyweight for $14 \mathrm{~d}$. It indicates that the drug $R N B$ might be helpful in the restoration of coagulating factors and in treating coagulation disorders. Our study results also showed that the APTT and DD were significantly less in Group B rats as compared to Group A rats after RNB intervention. But both were within their normal range. Therefore, because of the results of the above study and after interpretation of the results of our study, it can be said that $R N B$ might be helpful in the restoration of coagulation factors and can help treat the COVID patients.

CRP is a pentraxin found in most vertebrates (e. g.-mice, rats, and humans) and invertebrates such as the horseshoe crab (Limulus polyphemus). Human CRP (hCRP), discovered because it binds to pneumococcal C-polysaccharide (CPS), is an acute-phase protein, of which plasma levels can increase up to 1000 -fold following tissue damage or infection. In rats, CRP (rCRP) is not a typical acute-phase protein. However, in contrast to humans, rats have much higher plasma CRP concentrations under basal conditions, i.e. about 300$500 \mathrm{mg} / \mathrm{l}$, which is 100 times higher than the concentration in humans [26]. In our study, CRP evaluated by qualitative method was non-reactive in all rat groups, before and after RNB intervention which might be due to the use of the same reagents as used for evaluation of CRP in humans and there is a difference between rCRP and hCRP [26].

In the present study, the histopathological findings of both groups A and $B$ revealed no adverse effects on kidneys which is in agreement with the findings of a past study [27] that was carried out to evaluate the protective efficacy of silver nanoparticles synthesized from silymarin on cisplatin-induced oxidative stress in the albino rat. In that study, it was concluded that silver nanoparticles of silymarin protected the kidney of albino rats from the adverse effects caused by cisplatin [27].

The histological findings in the lungs of Group A and Group B rats of the present study are in agreement with the findings of a previous study [3] which was conducted to see the effects of GNPs on lung 
tissue of albino rats. The results of that work revealed severe alveolar damage in the form of collapsed alveoli, heavy infiltration in the lumen of alveoli, and interalveolar septa with inflammatory cells. These infiltrates are composed mainly of macrophages and associated with congested blood capillaries and extravasations of RBCs. This infiltration was severe near the bronchioles and blood vessels. The same finding was detected in other works in the form of perivascular and peribronchial neutrophilic and macrophage infiltration, shedding, and necrosis of the epithelium. These lesions were explained by oxidative damage induced by GNPs. This explanation was supported by the ability of GNPs to induce oxidative stress in bronchial epithelia. Furthermore, GNPs may cause damage to the epithelial cells leading to the damaged respiratory barrier. The changes like cellular infiltrations with extravasations of RBCs in the lung tissue as well as the chronic inflammatory infiltrate could be due to interference with the antioxidant defense mechanism, leading to the release of reactive oxygen species. Proteins and enzymes of lung tissue could also be affected [3].

The histological findings of the liver in-group ' $\mathrm{B}$ ' are more or less similar to the findings of a study conducted to investigate the particle-size effect of GNPs on the hepatic tissue. In this study, the exposure to GNPs doses produced alterations in the hepatocytes, portal triads, and sinusoids. The alterations in hepatocytes were hydropic degeneration, cloudy swelling, fatty degeneration, portal and lobular infiltrate by chronic inflammatory cells, and congestive dilated central veins. The induced histological alterations might be an indication of injured hepatocytes due to GNPs toxicity that became unable to deal with the accumulated residues resulting from metabolic and structural disturbances caused by these NPs [28].

The findings of no significant histological changes in liver, kidneys, and spleen after $R N B$ administration are in agreement with the findings of a previous study on mice [4], IP injected GNPs (12.5 nm, $40-400 \mu \mathrm{g} / \mathrm{kg}$ ) that were administered daily for 8 consecutive days were largely taken up by the spleen, kidney, and liver. There were no abnormal histopathological findings in the liver, kidney, and spleen after a 14-d repeated oral administration of GNPs or gold ions up to the dose of $1300 \mu \mathrm{g} / \mathrm{kg}$ [4]. In another study, it was reported that the repeated exposure of $14 \mathrm{~nm}$ GNPs for $56 \mathrm{~d}$ followed by a $14 \mathrm{~d}$ washout period showed their bioaccumulation without causing any structural or functional impairment in the liver and kidneys of the rats [29], which is supported by the findings of our study.

The histopathological findings of no major abnormality in different organs of our study are also in agreement with the histopathological findings of a previous study in which no abnormality was recorded concerning gross or histopathological examinations noted in PCC treated group of rats [12].

\section{CONCLUSION}

$R N B$ is having an immunomodulatory effect which is indicated by the increased percentage of monocytes in both groups $\mathrm{A}$ and $\mathrm{B}$ after $R N B$ intervention, indicating more number of macrophages and increased phagocytic activity. The results of coagulation tests indicate that the drug $R N B$ might be helpful in the restoration of coagulation factors and can help treat the COVID patients after doing human clinical trials.

The dose of $R N B$ plays an important role in their pathological effects on different organs of rats. The therapeutic dose of $R N B(26 \mathrm{mg} / \mathrm{kg}$ body weight) for a period of $14 \mathrm{~d}$ showed no harmful effect on lungs, liver, kidney, and spleen with the similar histological picture just like control group; however, after administration of 10 times higher dose of $R N B$ ( $260 \mathrm{mg} / \mathrm{kg}$ body weight) for a period of $14 \mathrm{~d}$, kidney and spleen showed no significant changes while it promotes inflammation and oxidative stress in liver and lungs tissue. Higher doses showed significant changes in the lungs and liver after activation of inflammatory response.

The activation of kupffer cells, an aggregate of more inflammatory cells in the liver and more inflammatory cell infiltrate within interalveolar septa, vacuolation, more lymphoid aggregates in interstitium in Group B rats after administration of higher dose (260 $\mathrm{mg} / \mathrm{kg}$ Bodyweight) is due to the protective mechanism of the body against $R N B$ but no major adverse effect of this drug is seen even after administration of 10 times higher dose as compared to the therapeutic dose. This drug is almost safe for the kidney and spleen even after administration of a higher dose. The findings of this experimental study of $R N B$ on Wistar rats may act as baseline data for planning further clinical trials in human study subjects to evaluate the effects on various comorbidities.

\section{ACKNOWLEDGEMENT}

The authors are grateful to the University's administration, Animal House staff, and technicians of the Central lab of UP University of Medical Sciences for their support in the completion of this study.

\section{THE SOURCE OF THE FUNDING}

This study was done under an intramural research project and its funding source was UP University of Medical Sciences, Saifai, Etawah, U. P., India. All authors had full access to all the data in the study and accept the responsibility to submit it for publication.

\section{AUTHORS CONTRIBUTIONS}

Dr. Amita Singh (Principal Investigator)-Literature search, study design, animal experiments, sample collection, figures, data collection, investigation, methodology, writing-original draft, visualization.

Prof. (Dr.) Raj Kumar (Co-Principal Investigator)-Conceptualization, funding acquisition, supervision, project administration, resources, validation.

Dr. Sanjay Kumar Kannaujia-Histopathological and Haematobiochemical investigations, literature search, photographs presentation, writing-review and editing.

Dr. Naresh Pal Singh-Statistical analysis, data interpretation, writingreview and editing, formal analysis.

\section{CONFLICT OF INTERESTS}

\section{Declared none}

\section{REFERENCES}

1. Kumar A, Singh A, Vishwakarma S, Gupta V. Raj Nirvan BatiWorld's $1^{\text {st }}$ Allovedic medicine effective in COVID-19 treatment. In: Covidology. UPUMS, Saifai, Jankipuram, Lucknow. Etawah: Savera Printing Press; 2020. p. 121-4.

2. Keerthana V, Dhanalakshmi S, Harikrishnan N. A perspective review on applications of nanoparticle mediated drug delivery to the cns. Int J Curr Pharm Sci. 2020;12(1):1-4. doi: 10.22159/ijcpr.2020v12i1.36819.

3. Elbakary RH, Okasha EF, Hassan Ragab AM, Ragab MH. Histological effects of gold nanoparticles on the lung tissue of adult male albino rats. J Microsc Ultrastruct. 2018;6(2):116-22. doi: 10.4103/JMAU.JMAU_25_18. PMID 30221136.

4. Ibrahim KE, Al-Mutary MG, Bakhiet AO, Khan HA. Histopathology of the liver, kidney, and spleen of mice exposed to gold nanoparticles. Molecules. 2018;23(8):1-14. doi: 10.3390/molecules23081848, PMID 30044410.

5. Kouadria M, Djemli S, Tahraoui A. The protective effect of zinc and magnesium against subchronic cadmium toxicity in wistar rats (Biochemical and neurobehavioral effects). Asian J Pharm Clin Res. 2019;12(5):217-25.

6. Long H, Nie L, Xiang X, Li H, Zhang X, Fu X, Ren H, Liu W, Wang $\mathrm{Q}, \mathrm{Wu} \mathrm{Q}$. D-dimer and prothrombin time are the significant indicators of severe COVID-19 and poor prognosis. BioMed Res Int. 2020;2020:6159720. doi: 10.1155/2020/6159720. PMID 32596339.

7. Yu HH, Qin C, Chen M, Wang W, Tian DS. D-dimer level is associated with the severity of COVID-19. Thromb Res. 2020;195:219-25. doi: 10.1016/j.thromres.2020.07.047, PMID 32777639.thromres.2020.07.047.

8. Singh A, Kumar R, Kannaujia SK Manikrishna, Singh NP. Immunohistochemical studies of the blood-brain barrier after administration of abhrakbhasm in wistar rats. Int J Sci Res. 2021;10(4):1-7. 
9. Shrestha P, Handral M. Evaluation of immunomodulatory activity of extract from rind of nephelium lappaceum fruit. Int J Pharm Pharm Sci. 2017;9Suppl 1:38-43.

10. Kumar R, Yadav R, Rawat R, Bajpai PK, Sharma IK, Kumar S. Role of raj Nirvan Bati (A novel ayurvedic preparation) in RTPCR positive COVID 19 cases: an interventional study. Ind J Med Sci Curr Res 2020;3Suppl 3:430-40.

11. Nair AB, Jacob S. A simple practice guide for dose conversion between animals and humans. J Basic Clin Pharma. 2016;7(2):7Spppl2:27-31. doi: 10.4103/0976-0105.177703.

12. Chitra B, Ramaswamy RS, Suba V. Toxicity evaluation of purna cantirotaya centuram, a siddha medicine in wistar rats. Int Sch Res Not. 2015;1-10.

13. Buncharoen W, Saenphet S, Chomdej S, Saenphet K. Evaluation of biochemical, hematological, and histopathological parameters of albino rats treated with stemonaaphylla craib. Extract J Med Plants Res. 2012;6(27):4429-35.

14. Nfambi J, Bbosa GS, Sembajwe LF, Gakunga J, Kasolo JN. Immunomodulatory activity of methanolic leaf extract of moringa oleifera in wistar albino rats. J Basic Clin Physiol Pharmacol. 2015;26(6)Suppl 6:603-11. doi: 10.1515/jbcpp2014-0104, PMID 26103628.

15. Bhardwaj KR, Upadhyay DS. Blood collection in laboratory animals. Laboratory animals for research. NLAC. Lucknow, India; 1994. p. 30.

16. Rats. Who's certified [internet]. WI: Clinical Laboratory Parameters for crl (Han). Charles River Laboratories; 2008. Available from: https://www.criver.com [Last accessed on 06 Jul 2020]

17. Garcia Manzano A, Gonzalez Llaven J, Lemini C, Rubio Poo C. Standardization of rat blood clotting tests with reagents used for humans. Proc West Pharmacol Soc. 2001;44:153-5. PMID 11793967.

18. Mayne P, Mayne PD. Clinical chemistry in diagnosis and treatment. 6th ed A Hodder Arnold Publication. London, UK; 1994.

19. Manogaran M, Ganeshan J. In vivo ameliorative potential of cafeic acid against hepatotoxicity and nephrotoxicity induced by mercuric chloride in albino wistar rats. Asian J Pharm Clin Res. 2019;12(4):77-83.

20. Mandal A. What is a macrophage? [internet]. News-medical 2019. Available from: https://www.news-medical.net/life-
sciences/What is-a-Macrophage.aspx. [Last accessed on 05 Jul 2021]

21. Tang N, Li D, Wang X, Sun Z. Abnormal coagulation parameters are associated with poor prognosis in patients with novel coronavirus pneumonia. J Thromb Haemost. 2020;18(4):844-7. doi: 10.1111/jth.14768. PMID 32073213.

22. Han W, Quan B, Guo Y, Zhang J, Lu Y, Feng G, Wu Q, Fang F, Cheng L, Jiao N, Li X, Chen Q. The course of clinical diagnosis and treatment of a case infected with coronavirus disease 2019. J Med Virol. 2020;92(5) Suppl 5:461-3. doi: 10.1002/jmv.25711, PMID 32073161.

23. Tang N, Bai H, Chen X. Anticoagulant treatment is associated with decreased mortality in severe coronavirus disease 2019 patients with coagulopathy. J Thromb Haemost. 2020;18(5):1094-9. doi: 10.1111/jth.14817.

24. Li XY, Du B, Wang YS. The key points in the treatment of the critical coronavirus disease 2019 patient. Zhonghua Jie $\mathrm{He} \mathrm{He}$ Hu Xi Za Zhi. 2020;43:E026. doi: 10.3760/cma.j.cn11214720200224-00159.

25. Zhou F, Yu T, Du R, Fan G, Liu Y, Liu Z, Xiang J, Wang Y, Song B, Gu X, Guan L, Wei Y, Li H, Wu X, Xu J, Tu S, Zhang Y, Chen H, Cao B. Clinical course and risk factors for mortality of adult inpatients with COVID-19 in Wuhan, China: a retrospective cohort study. Lancet. 2020;395(10229):1054-62. doi: 10.1016/S0140-6736(20)30566-3, PMID 32171076.

26. Padilla ND, Bleeker WK, Lubbers Y, Rigter GMM, van Mierlo GJ, Daha MR, Hack CE Rat C-reactive protein activates the autologous complement system. Immunology. 2003;109(4):564-71. doi: 10.1046/j.1365-2567.2003.01681.x.

27. Janakiraman M. Protective efficacy of silver nanoparticles synthesized from silymarin on cisplatin-induced renal oxidative stress in the albino rat. Int J Appl Pharm. 2018;10(5):110-6.

28. Abdelhalim MA, Jarrar BM. Histological alterations in the liver of rats induced by different gold nanoparticle sizes, doses and exposure duration. J Nanobiotechnology. 2012;10:5. doi: 10.1186/1477-3155-10-5. PMID 22276919.

29. Rambanapasi C, Zeevaart JR, Buntting H, Bester C, Kotze D, Hayeshi $\mathrm{R}$, Grobler A. Bioaccumulation and subchronic toxicity of 14-nm gold nanoparticles in rats. Molecules. 2016;21(6):21.763. doi: 10.3390/molecules21060763, PMID 27294904. 